\title{
Three-Dimensional Dendritic Cu-Co-P Electrode by One-Step Electrodeposition on a Hydrogen Bubble Template for Hydrogen Evolution Reaction
}

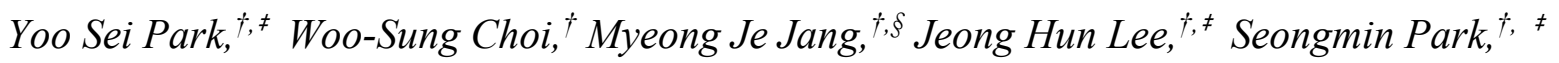
Hyunsoo Jin,,$^{\neq}$Min Ho Seo, " Kyu-Hwan Lee, ${ }^{\dagger}$, Yadong Yin, ${ }^{\perp}$ Yangdo Kim, ${ }^{*, \neq}$ Juchan Yang, ${ }^{*}+$ Sung Mook Choi ${ }^{*},+$

$\dagger$ Materials Center for Energy Department, Surface Technology Division, Korea Institute of Materials Science, 797 Changwondaero, Seongsangu, Gyeongnam, 51508, Republic of Korea

Department of Materials Science and Engineering, Pusan National University, 2 Busandaehak-ro 63beon-gil, Geumjeong-gu, Busan, 46241, Republic of Korea

$\S$ Advanced Materials Engineering, Korea University of Science and Technology (UST), 217 Gajeong-ro, Yuseong-gu, Daejeon, 34113, Republic of Korea

"Hydrogen and Fuel Cell Center, New and Renewable Energy Institute, Korea Institute of Energy Research, 20-41 Sinjaesaengeneoji-ro, Haseo-myeon, Buan-gun, Jeollabuk-do, 56332, Republic of Korea

${ }^{\perp}$ Department of Chemistry, Materials Science and Engineering program and UCR Center for Catalysis University of California Riverside, 501 Big springs Rd, CA 92521, USA.

Number of page: 17

Number of table: 1

Number of figure: 13

*To whom correspondence should be addressed.

* E-mail: yangdo@pusan.ac.kr

* E-mail: juchana83@kims.re.kr

*E-mail: akyzaky@,kims.re.kr 

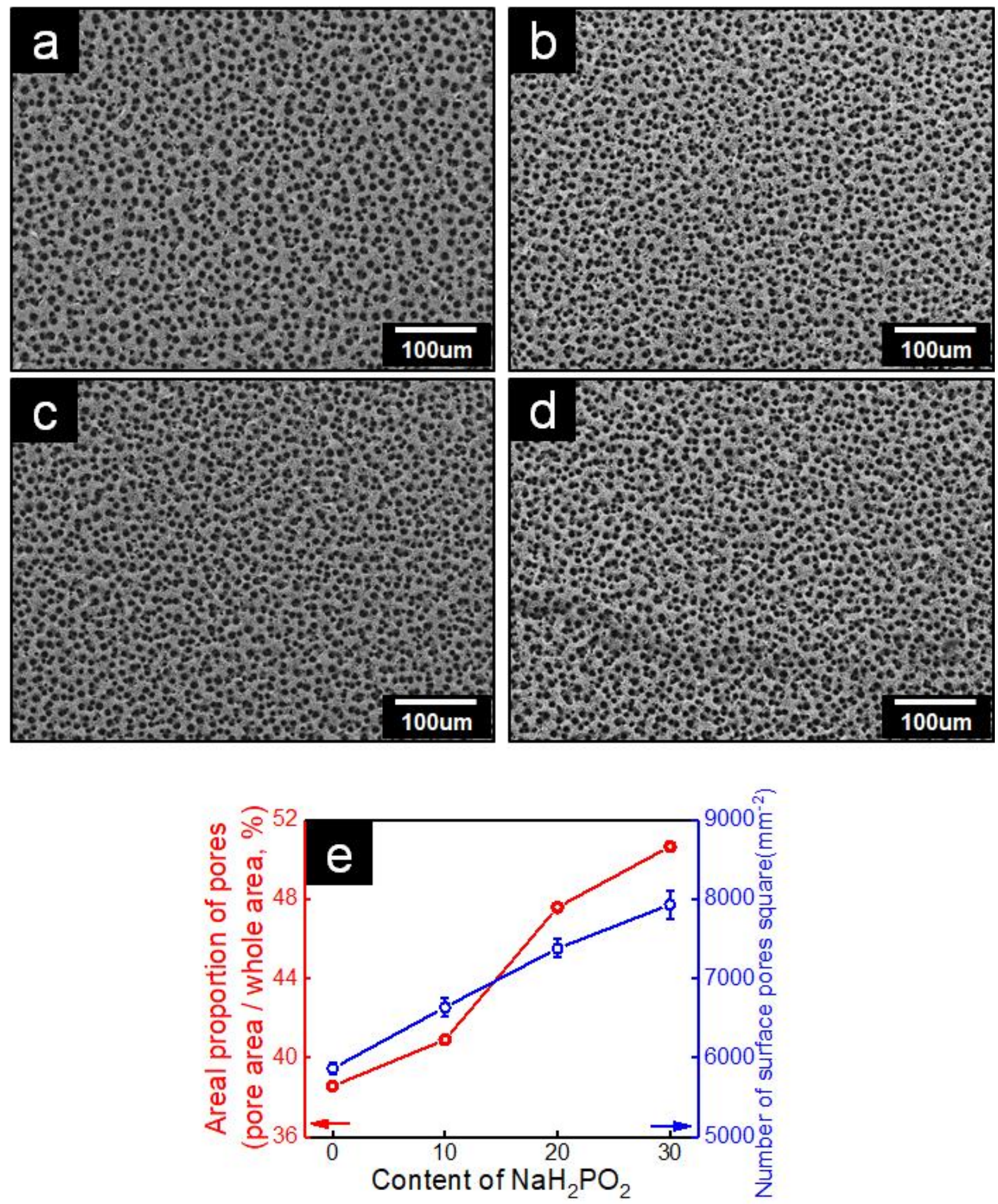

Figure S1. SEM image for proportion of pores with different $\mathrm{NaH}_{2} \mathrm{PO}_{2}$ : (a) $0 \mathrm{mM}$, (b) $10 \mathrm{mM}$, (c) $20 \mathrm{mM}$ and (d) $30 \mathrm{mM}$. (e) The areal proportion of pores and number of surface pores. 

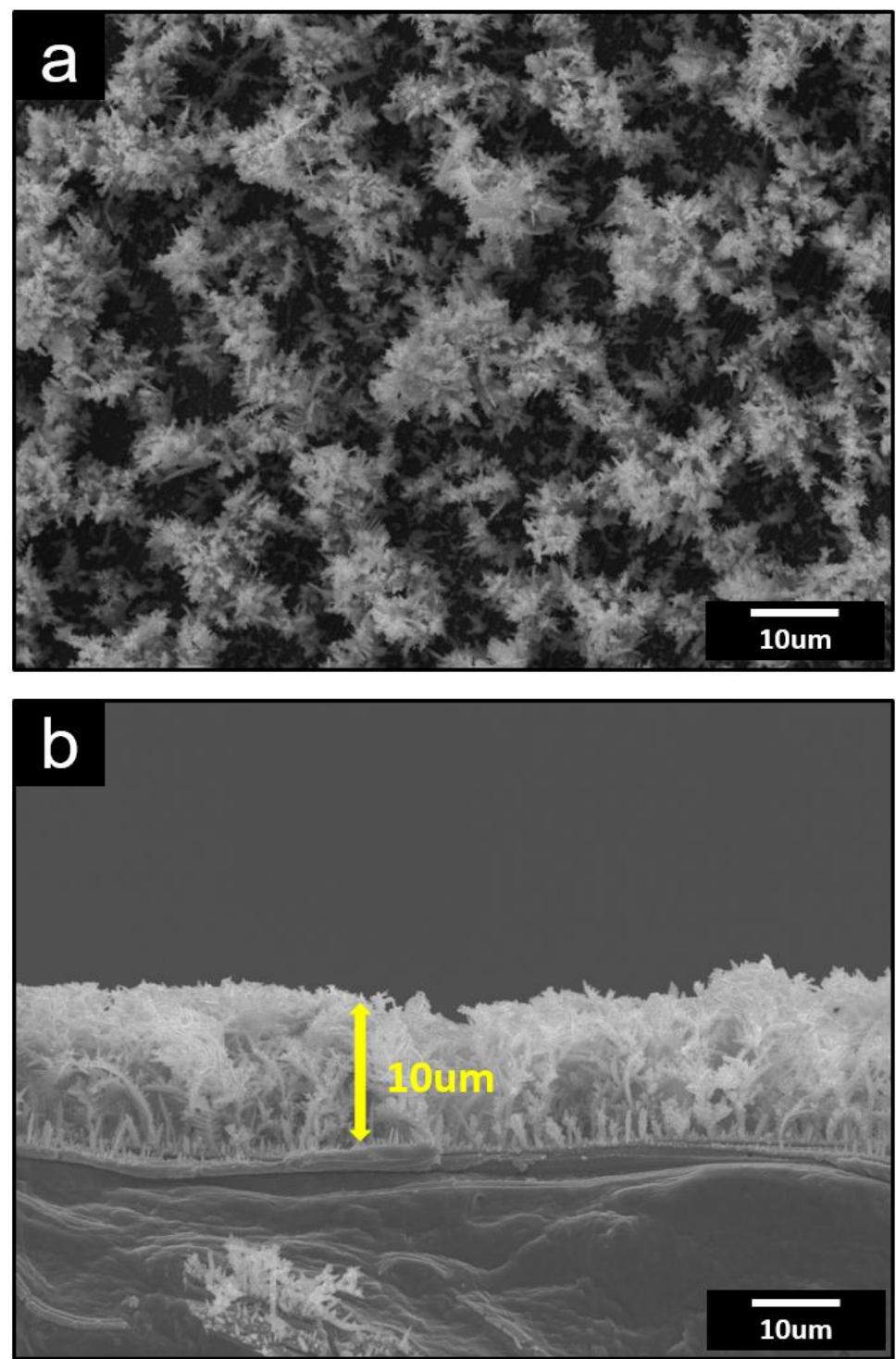

Figure S2. The SEM image of (a) top view and (b) cross-section images for $\mathrm{Cu}-\mathrm{Co}-\mathrm{P}$ foam fabricated at $40 \mathrm{mM} \mathrm{NaH}{ }_{2} \mathrm{PO}_{2}$ in electrodeposition solution. 

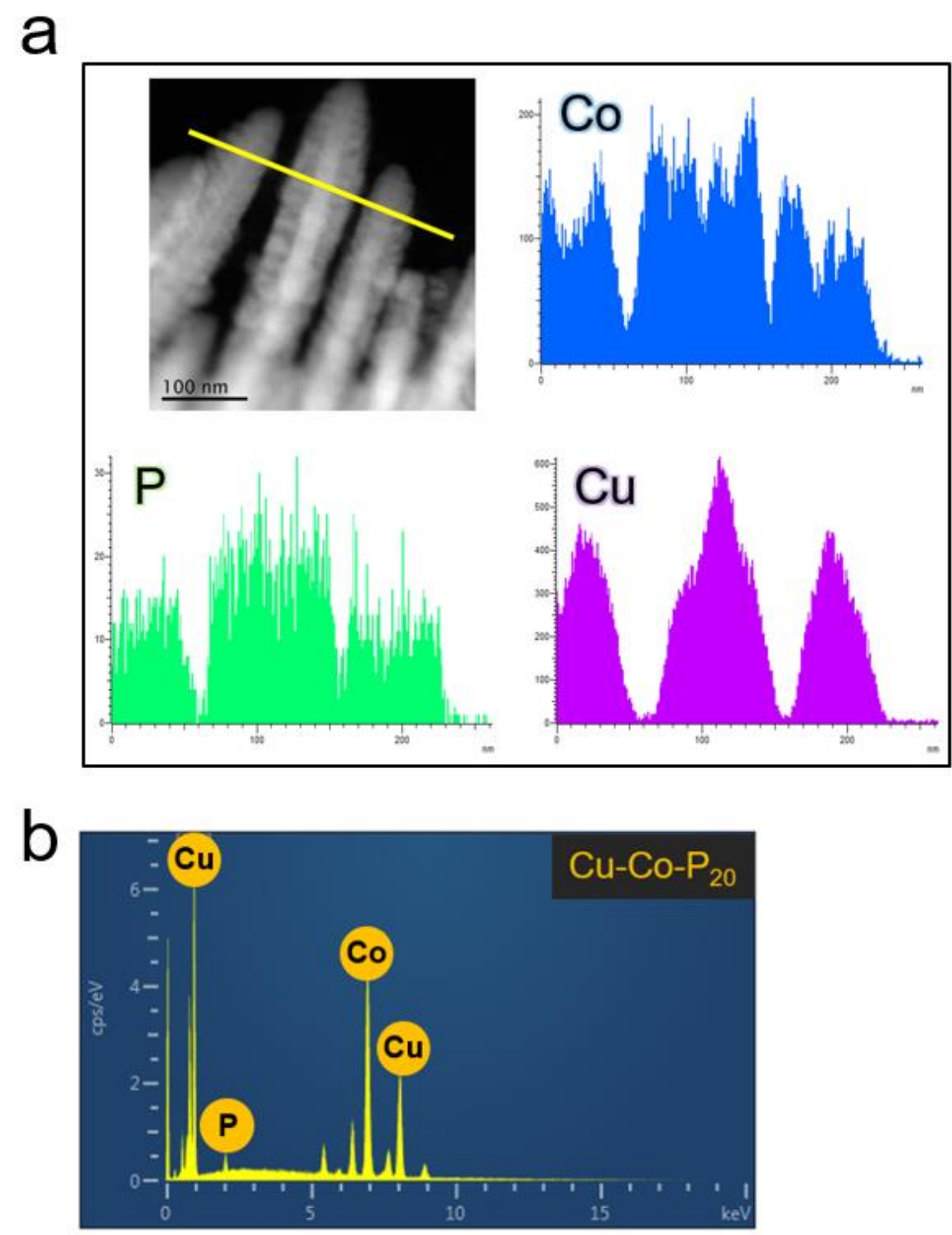

Figure S3. TEM images of the $\mathrm{Cu}-\mathrm{Co}-\mathrm{P}_{20}$ foam and EDS (Energy dispersive spectrometer) analysis. (a) The line profile results of $\mathrm{Cu}, \mathrm{Co}$ and $\mathrm{P}$ along the line drawing across the diameter of a thorn region in dendritic structure. (b) EDS full spectra of dendritic structure of Cu-Co$\mathrm{P}_{20}$. 


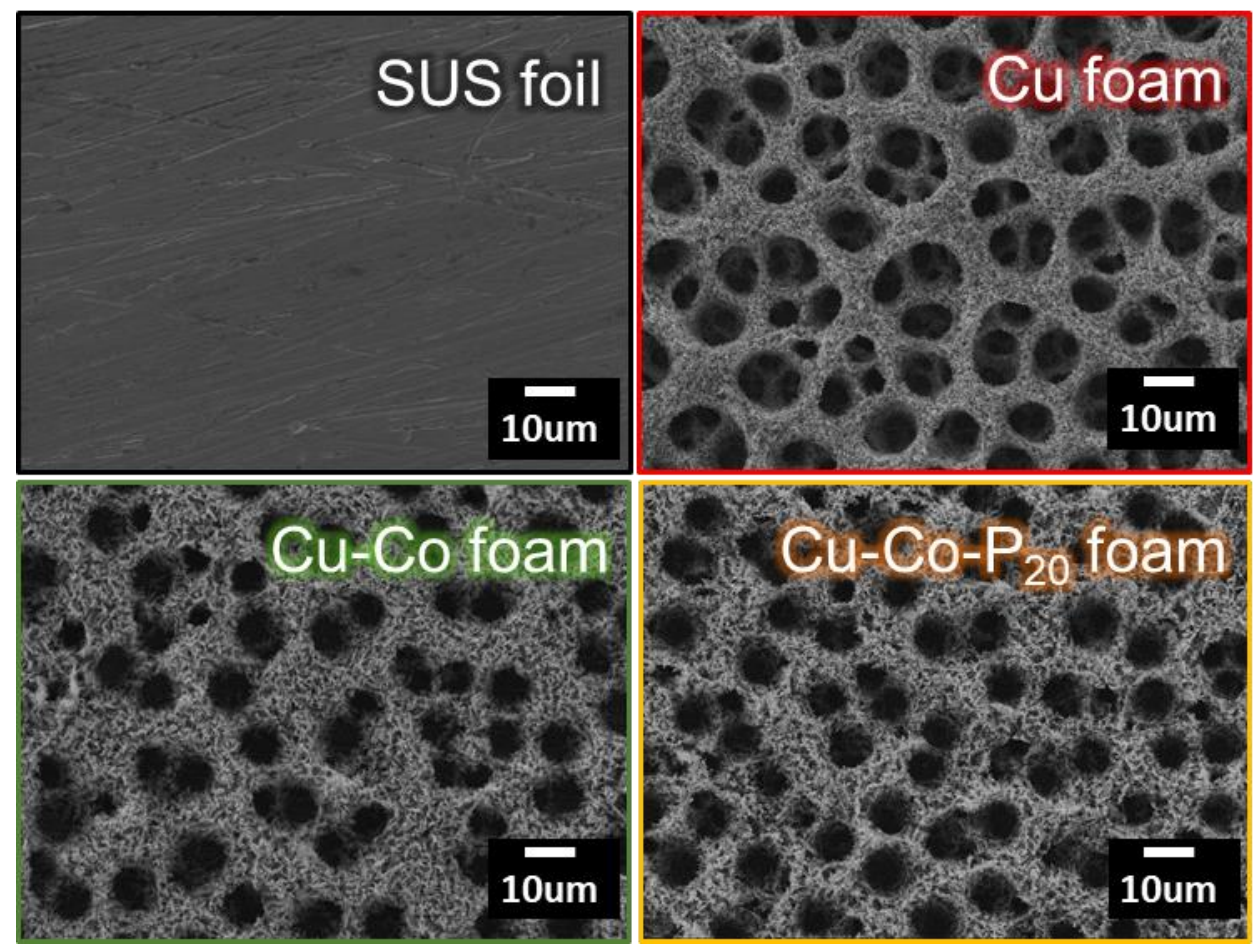

Figure S4. SEM images of the SUS-foil, $\mathrm{Cu}$-foam, $\mathrm{Cu}-\mathrm{Co}$ foam and $\mathrm{Cu}-\mathrm{Co}-\mathrm{P}_{20}$ foam in $\mathrm{N}_{2}-$ saturated $1 \mathrm{M} \mathrm{KOH}$ solution. 


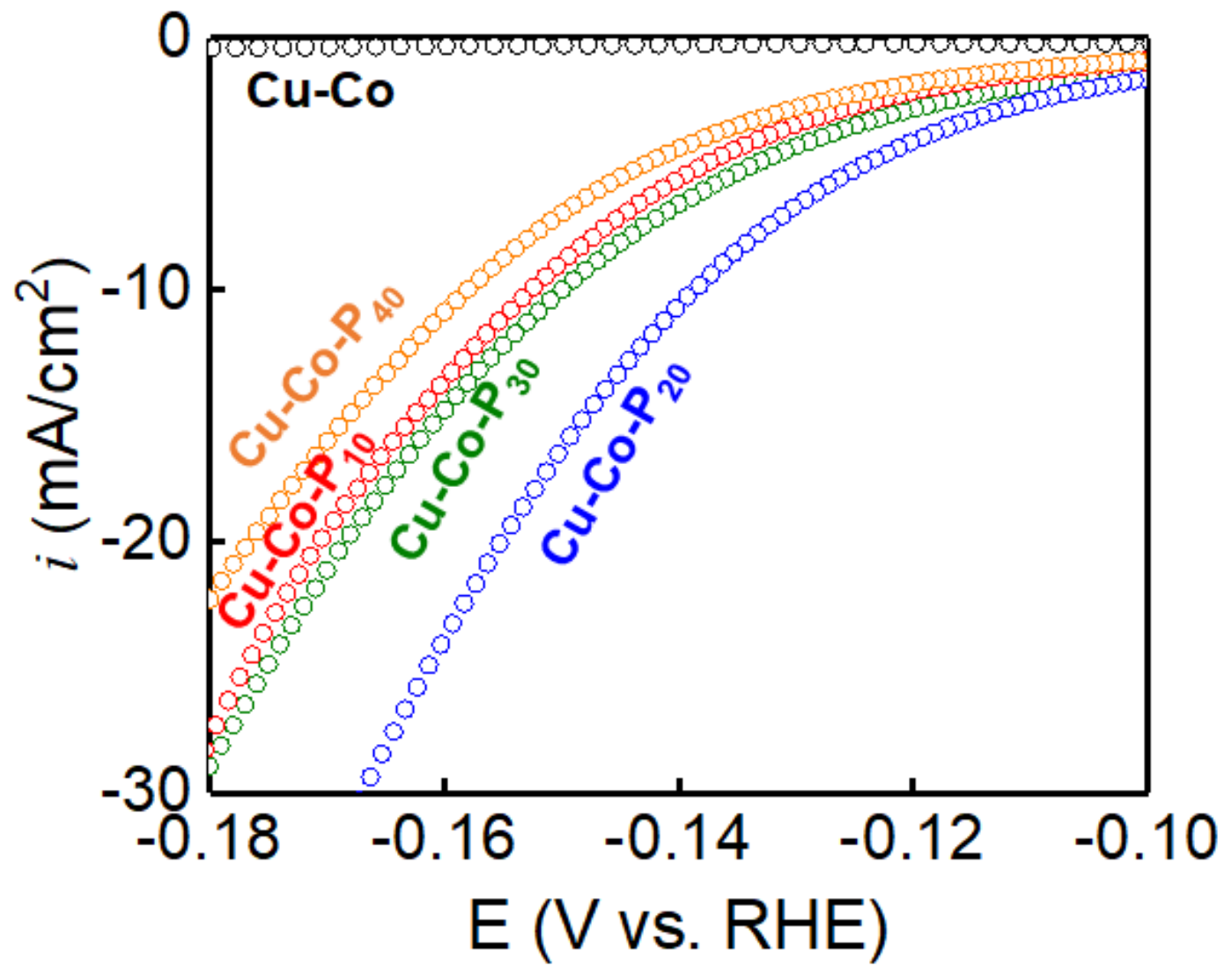

Figure S5. Polarization curves of $\mathrm{Cu}-\mathrm{Co}-\mathrm{P}_{\mathrm{x}}$ foams with different concentration of $\mathrm{NaH}_{2} \mathrm{PO}_{2}$ from HER polarizations(fig. 3b). 

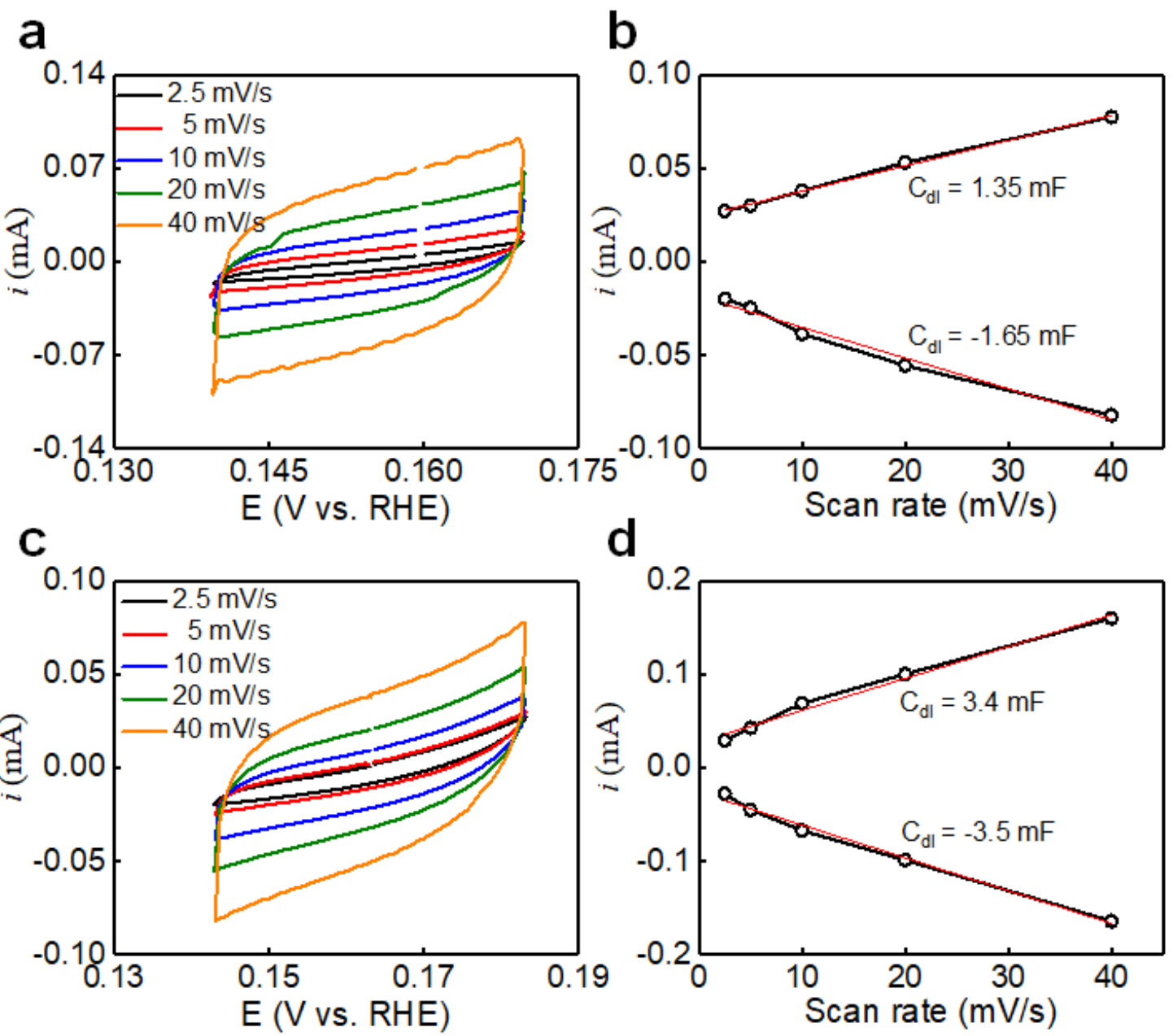

Figure S6. Electrochemical surface area analysis. (a, c) Cyclic voltammetry curves of Cu-Co and (b) $\mathrm{Cu}-\mathrm{Co}-\mathrm{P}_{20}$ foams in a non-faradaic region in $1 \mathrm{M} \mathrm{KOH}$ at different scan rate. (b, d) Current versus scan rate plots for (c) $\mathrm{Cu}-\mathrm{Co}$ and (d) $\mathrm{Cu}-\mathrm{Co}-\mathrm{P}_{20}$ foams (refer to Figure 4c). 

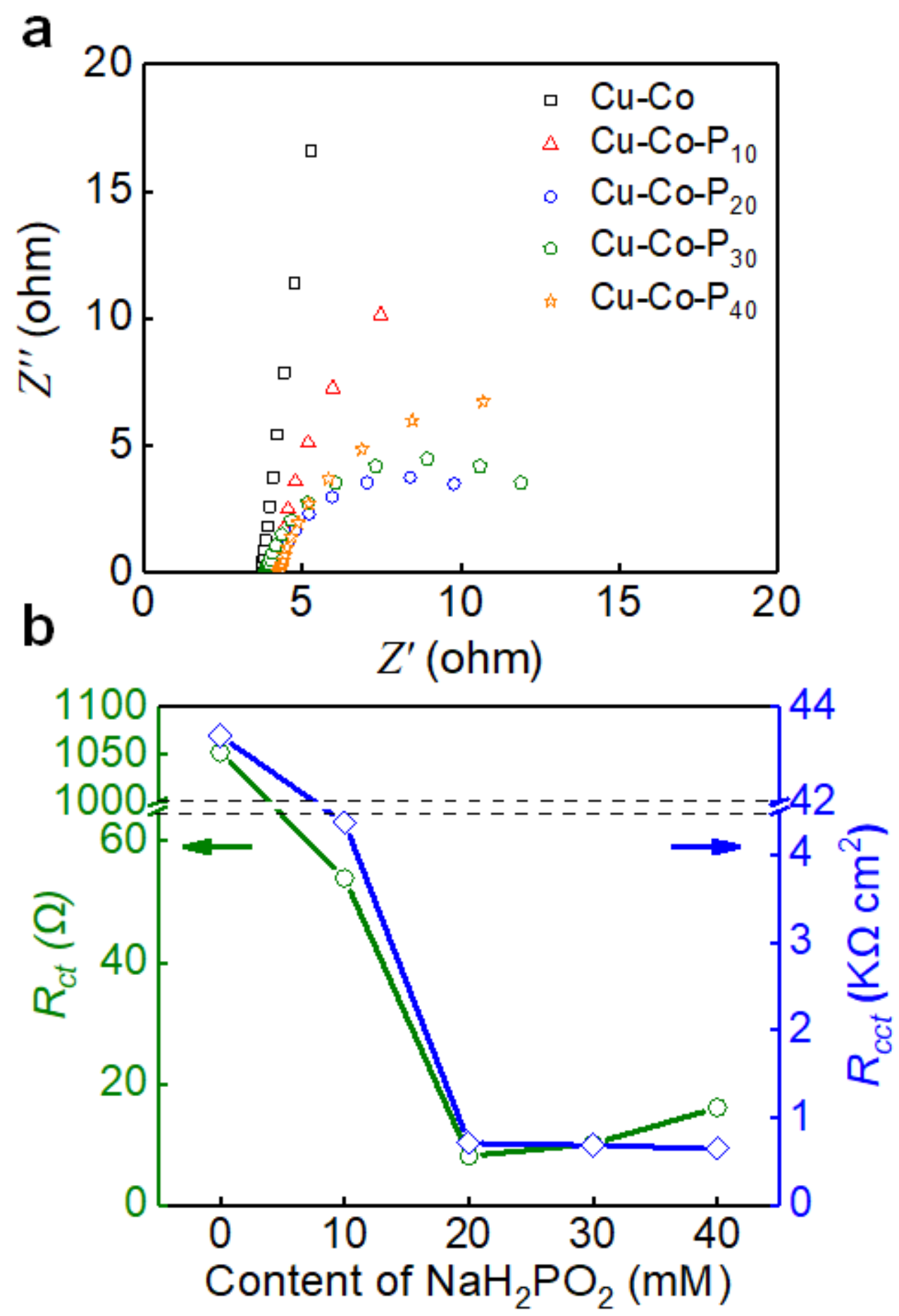

Figure S7. (a) Electrochemical impedance spectra of $\mathrm{Cu}-\mathrm{Co}$ and $\mathrm{Cu}-\mathrm{Co}-\mathrm{P}_{\mathrm{x}}(\mathrm{x}=10,20,30$ and 40) foams at $-150 \mathrm{mV}$ vs. RHE in $1 \mathrm{M} \mathrm{KOH} \mathrm{(b)} \mathrm{charge} \mathrm{transfer} \mathrm{resistance}\left(R_{c t}\right)$ and surface areacorrected charge transfer resistance $\left(R_{\mathrm{cct}}\right)$ at different content of $\mathrm{NaH}_{2} \mathrm{PO}_{2}\left(R_{\mathrm{cct}}=\mathrm{ECSA} \times R_{\mathrm{ct}}\right)$ 


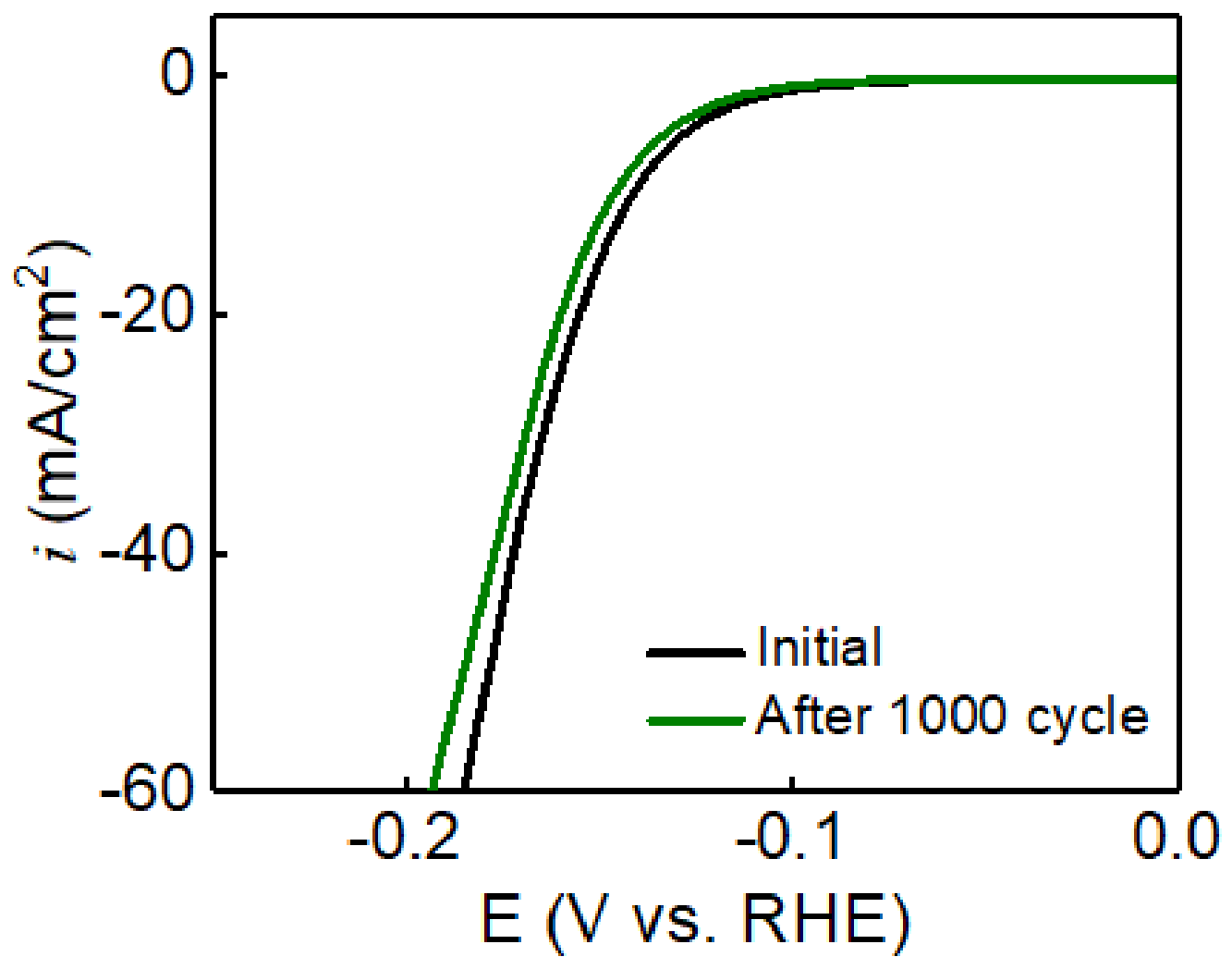

Figure S8. HER durability test of $\mathrm{CuCoP}_{20}$ foam. Catalysts were polarized repeatedly in $\mathrm{N}_{2}$ purged $1 \mathrm{M} \mathrm{KOH}(\mathrm{aq})$. Polarization curves at the $1^{\text {st }}$ (black line) and $1000^{\text {th }}$ (green line) cycle were selectively demonstrated. 
a

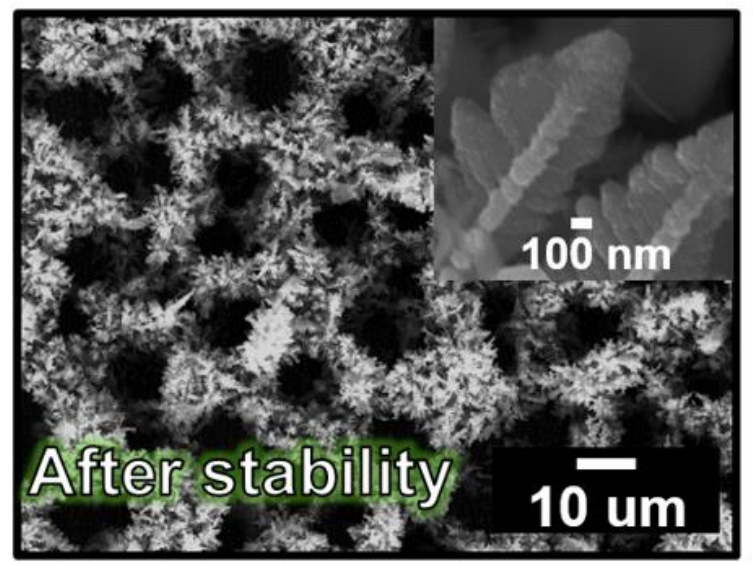

b
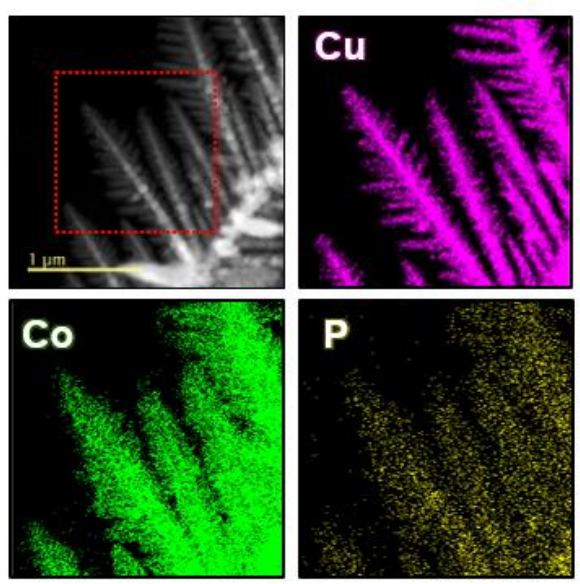

Figure S9. (a) SEM images $\mathrm{Cu}-\mathrm{Co}-\mathrm{P}_{20}$ foam and (b) TEM-EDS elemental mapping images of $\mathrm{Co}$ (green), $\mathrm{Cu}$ (purple) and $\mathrm{P}\left(\right.$ yellow) in dendritic $\mathrm{Cu}-\mathrm{Co}-\mathrm{P}_{20}$ foam after 1000 cycle of LSV. 


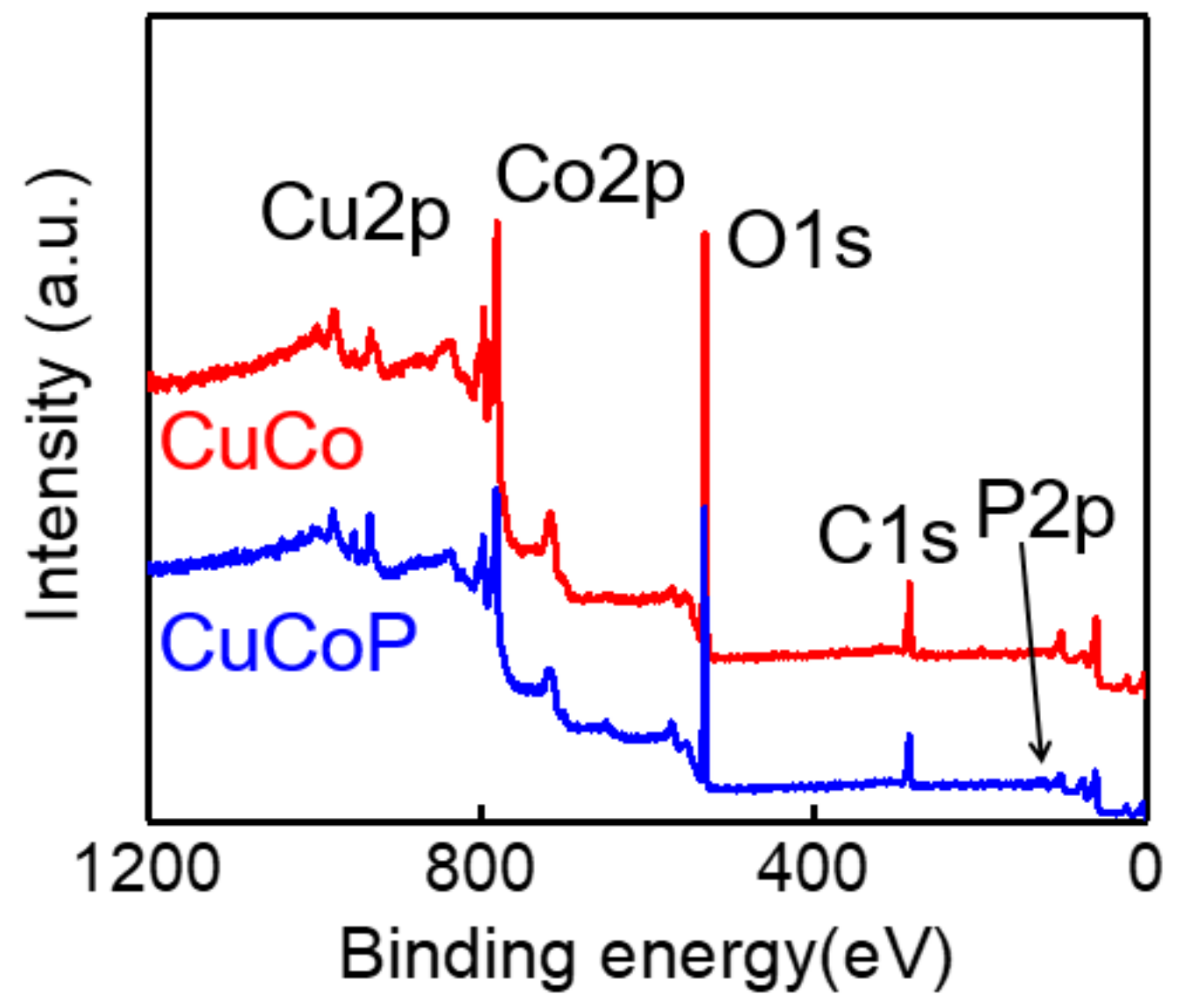

Figure S10. XPS survey spectrum of $\mathrm{Cu}-\mathrm{Co}$ and $\mathrm{Cu}-\mathrm{Co}-\mathrm{P}_{20}$ foams. 

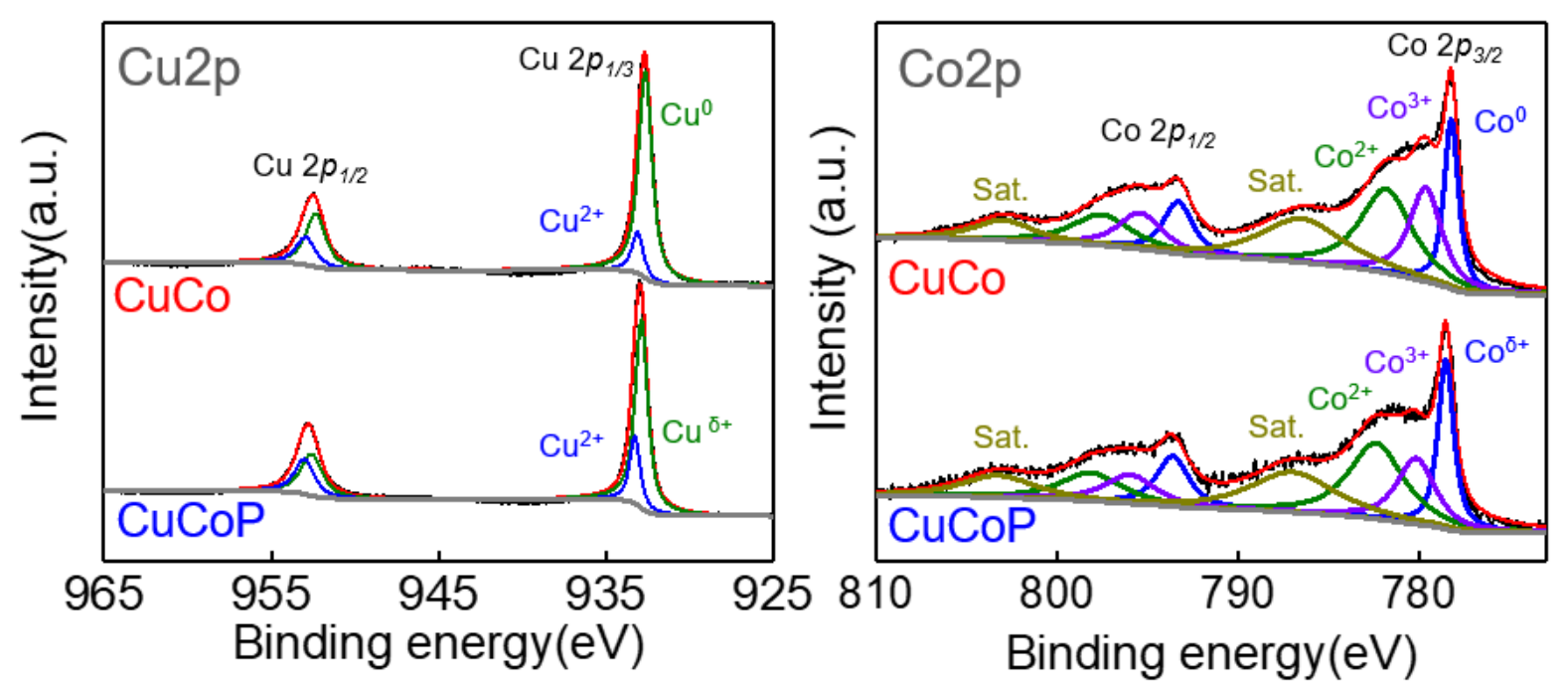

Figure S11. The deconvoluted high resolution XPS spectra of $\mathrm{Cu} 2 \mathrm{p}$ and $\mathrm{Co} 2 \mathrm{p}$ of $\mathrm{Cu}-\mathrm{Co}-\mathrm{P}_{20}$ foams. 

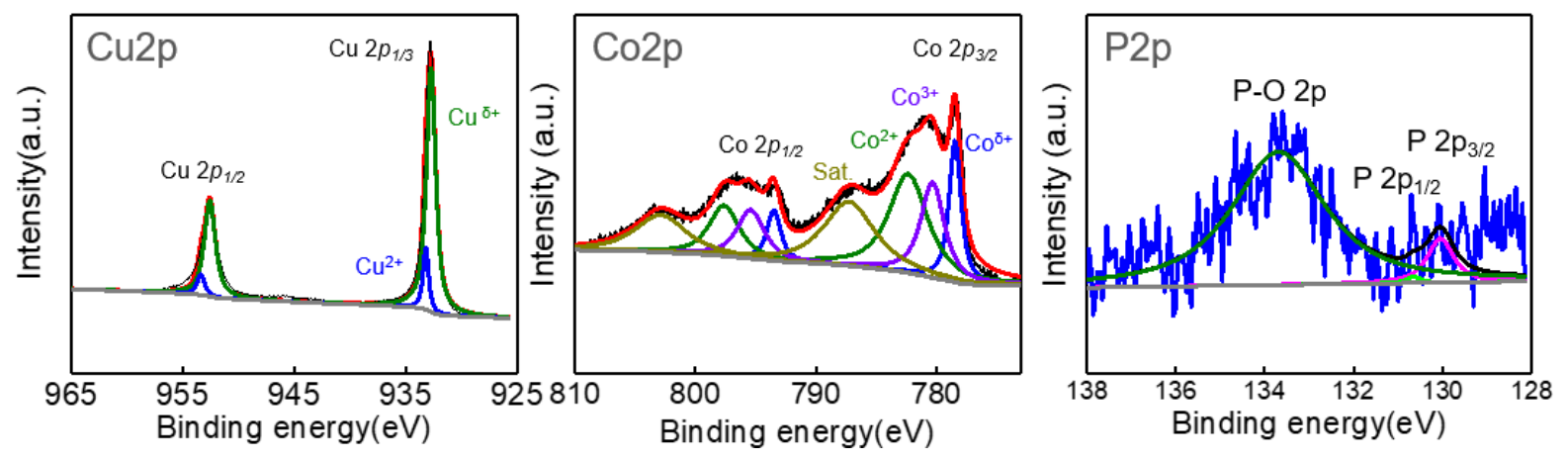

Figure S12. The high-resolution core level XPS spectra of $\mathrm{Cu} 2 p, \mathrm{Co} 2 p$ and P $2 p$ of Cu-Co-

$\mathrm{P}_{20}$ foams after durability test. 

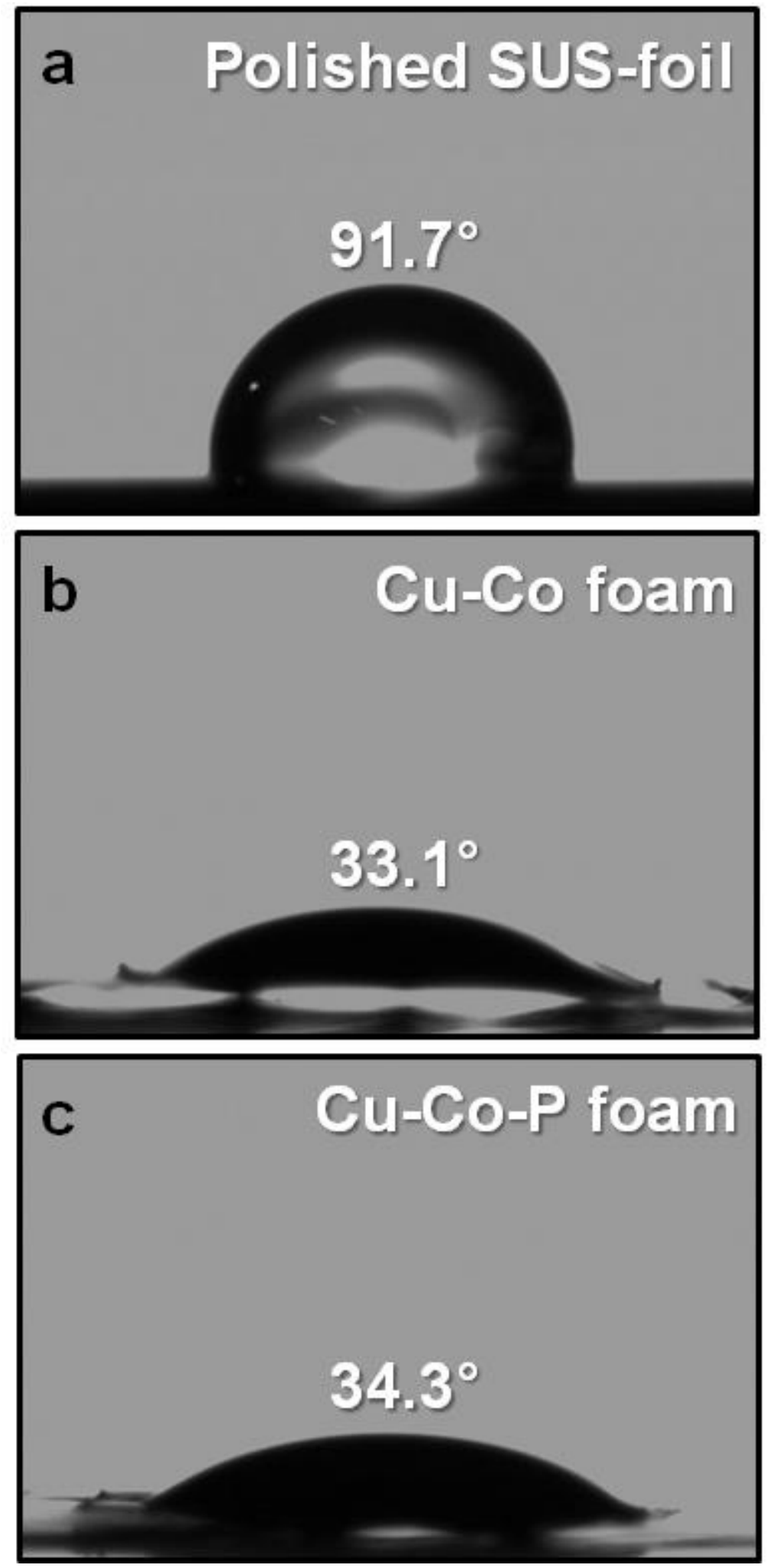

Figure S13. Wettability. The contact angle of (a) polished SUS-foil, (b) Cu-Co foam and (c) $\mathrm{Cu}-\mathrm{Co}-\mathrm{P}_{20}$ foams. 
Table S1. Comparison of the HER activities of non-noble metal catalysts in alkaline solutions in terms of overpotential and tafel slope.

\begin{tabular}{|c|c|c|c|c|c|c|}
\hline Electrode & \multicolumn{2}{|c|}{$\begin{array}{l}\text { Over-potential } \\
\left(\text { at } 10 \mathrm{~mA} / \mathrm{cm}^{2}\right)\end{array}$} & $\begin{array}{c}\text { iR- } \\
\text { correction }\end{array}$ & $\begin{array}{l}\text { Tafel } \\
\text { slope }\end{array}$ & Solution & Reference \\
\hline \multirow{2}{*}{$\begin{array}{l}\text { CoCuP foam } \\
\text { on SUS-foil }\end{array}$} & 10 & 138 & \multirow[t]{2}{*}{ O (85\%) } & \multirow[t]{2}{*}{48} & \multirow[t]{2}{*}{$1 \mathrm{M} \mathrm{KOH}$} & \multirow[t]{2}{*}{ This work } \\
\hline & 20 & 154 & & & & \\
\hline $\begin{array}{c}\mathrm{Co}_{2} \mathrm{P} \\
\text { on Co-foil }\end{array}$ & 10 & 154 & $x$ & 59 & $1 \mathrm{M} \mathrm{KOH}$ & [1] \\
\hline $\begin{array}{c}\mathrm{Co} / \mathrm{CoP} \\
\text { on } \mathrm{N} \text {-doped } \\
\text { carbon }\end{array}$ & 10 & 138 & $O\left({ }^{*}\right)$ & 64 & $1 \mathrm{M} \mathrm{KOH}$ & [2] \\
\hline $\begin{array}{c}\mathrm{Co}_{2} \mathrm{P} \\
\text { on Si-wafer }\end{array}$ & 10 & 183 & $x$ & 66 & $1 \mathrm{M} \mathrm{KOH}$ & [3] \\
\hline $\begin{array}{c}\mathrm{Cu}_{3} \mathrm{P} \\
\text { on copper-mesh }\end{array}$ & 10 & 252 & $x$ & 150 & $1 \mathrm{M} \mathrm{KOH}$ & [4] \\
\hline $\begin{array}{c}\text { CoP } \\
\text { on carbon-cloth }\end{array}$ & 10 & 209 & $x$ & 129 & $1 \mathrm{M} \mathrm{KOH}$ & [5] \\
\hline $\begin{array}{c}\mathrm{Cu}_{3} \mathrm{P}-\mathrm{CoP} \\
\text { on carbon-cloth }\end{array}$ & 20 & 208 & $\mathrm{O}\left({ }^{*}\right)$ & * & $1 \mathrm{M} \mathrm{KOH}$ & [6] \\
\hline $\begin{array}{c}\mathrm{Cu}_{3} \mathrm{P} \\
\text { on Cu-mesh }\end{array}$ & 10 & 266 & $O\left({ }^{*}\right)$ & 107 & $1 \mathrm{M} \mathrm{KOH}$ & [7] \\
\hline $\begin{array}{c}\text { CoP } \\
\text { on N-doped } \\
\text { carbon }\end{array}$ & 10 & 129 & $\mathrm{O}\left({ }^{*}\right)$ & 58 & $1 \mathrm{M} \mathrm{KOH}$ & [8] \\
\hline $\begin{array}{l}\text { CuCoP } \\
\text { on Cu-foil }\end{array}$ & 10 & 231 & $\mathrm{O}(60 \%)$ & 86 & $1 \mathrm{M} \mathrm{KOH}$ & [9] \\
\hline $\begin{array}{c}\text { CoP } \\
\text { on GCE }\end{array}$ & 10 & 175 & $O\left({ }^{*}\right)$ & 84 & $1 \mathrm{M} \mathrm{KOH}$ & [10] \\
\hline
\end{tabular}

O*: IR-corrected data but, not mentioned the value in paper

Table S1. Comparison of the HER activities of non-noble metal catalysts in alkaline solutions in terms of overpotential and tafel slope. 


\section{Supporting Ref.}

1. Yuan, C.-Z.; Zhong, S.-L.; Jiang, Y.-F.; Yang, Z. K.; Zhao, Z.-W.; Zhao, S.-J.; Jiang, N.; Xu, A.-W., Direct growth of cobalt-rich cobalt phosphide catalysts on cobalt foil: an efficient and self-supported bifunctional electrode for overall water splitting in alkaline media. Journal of Materials Chemistry A 2017, 5 (21), 10561-10566, DOI 10.1039/C7TA01776F.

2. Wang, H.; Min, S.; Wang, Q.; Li, D.; Casillas, G.; Ma, C.; Li, Y.; Liu, Z.; Li, L.-J.; Yuan, J.; Antonietti, M.; Wu, T., Nitrogen-Doped Nanoporous Carbon Membranes with Co/CoP Janus-Type Nanocrystals as Hydrogen Evolution Electrode in Both Acidic and Alkaline Environments. ACS Nano 2017, 11 (4), 4358-4364, DOI 10.1021/acsnano.7b01946.

3. Read, C. G.; Callejas, J. F.; Holder, C. F.; Schaak, R. E., General Strategy for the Synthesis of Transition Metal Phosphide Films for Electrocatalytic Hydrogen and Oxygen Evolution. ACS Applied Materials \& Interfaces 2016, 8 (20), 12798-12803, DOI 10.1021/acsami.6b02352.

4. Wei, S.; Qi, K.; Jin, Z.; Cao, J.; Zheng, W.; Chen, H.; Cui, X., One-Step Synthesis of a Self-Supported Copper Phosphide Nanobush for Overall Water Splitting. ACS Omega 2016, 1 (6), 1367-1373, DOI 10.1021/acsomega.6b00366.

5. Tian, J.; Liu, Q.; Asiri, A. M.; Sun, X., Self-Supported Nanoporous Cobalt Phosphide Nanowire Arrays: An Efficient 3D Hydrogen-Evolving Cathode over the Wide Range of pH 0-14. Journal of the American Chemical Society 2014, 136 (21), 7587-7590, DOI 10.1021/ja503372r.

6. Du, H.; Zhang, X.; Tan, Q.; Kong, R.; Qu, F., A Cu3P-CoP hybrid nanowire array: a superior electrocatalyst for acidic hydrogen evolution reactions. Chemical Communications 2017, 53 (88), 12012-12015, DOI 10.1039/C7CC07802A. 
7. Wang, H.; Zhou, T.; Li, P.; Cao, Z.; Xi, W.; Zhao, Y.; Ding, Y., Self-Supported Hierarchical Nanostructured NiFe-LDH and Cu3P Weaving Mesh Electrodes for Efficient Water Splitting. ACS Sustainable Chemistry \& Engineering 2018, 6 (1), 380-388, DOI 10.1021/acssuschemeng. $7 \mathrm{~b} 02654$.

8. Ma, J.; Wang, M.; Lei, G.; Zhang, G.; Zhang, F.; Peng, W.; Fan, X.; Li, Y., Polyaniline Derived N-Doped Carbon-Coated Cobalt Phosphide Nanoparticles Deposited on N-Doped Graphene as an Efficient Electrocatalyst for Hydrogen Evolution Reaction. Small 2018, 14 (2), 1702895, DOI 10.1002/smll.201702895.

9. Wasalathanthri, R. N.; Jeffrey, S.; Awni, R. A.; Sun, K.; Giolando, D. M., Electrodeposited Copper-Cobalt-Phosphide: A Stable Bifunctional Catalyst for Both Hydrogen and Oxygen Evolution Reactions. ACS Sustainable Chemistry \& Engineering 2019, 7 (3), 3092-3100, DOI 10.1021/acssuschemeng.8b04807.

10. Sumboja, A.; An, T.; Goh, H. Y.; Lübke, M.; Howard, D. P.; Xu, Y.; Handoko, A. D.; Zong, Y.; Liu, Z., One-Step Facile Synthesis of Cobalt Phosphides for Hydrogen Evolution Reaction Catalysts in Acidic and Alkaline Medium. ACS Applied Materials \& Interfaces 2018, 10 (18), 15673-15680, DOI 10.1021/acsami.8b01491. 